\title{
Enabling integrated modelling systems for environmental prediction at national scale
}

\author{
$\underline{\text { A. Specht }}^{\mathrm{a}} \odot$, S.M. Guru ${ }^{\mathrm{a}} \odot$ and B. Morris ${ }^{\mathrm{a}} \odot$ \\ ${ }^{a}$ Terrestrial Ecosystem Research Network (TERN), University of Queensland. \\ email: a.specht@uq.edu.au
}

\begin{abstract}
As clearly established in a series of discussion papers commissioned by the Australian government, there is an urgent need for improved ability to integrate the wealth of available data and modelling expertise to enable flexible and timely prediction of future environmental scenarios. The Australian government, under the National Collaborative Research Infrastructure Strategy (NCRIS), has established a robust research infrastructure $^{1}$ for the organisation of data and information across many environmental domains. This has enabled unprecedented access to systematically collected and curated high-quality data. As a result, some limited, largely domain-specific predictive capacity has developed, but active and continuing cross-domain synthesis is required for effective responses to the multi- and trans-disciplinary challenges facing us, such as the threats posed by climate change and other environmental hazards associated with breaching our planetary health boundaries.
\end{abstract}

In this paper we present an approach to establish a cross-domain, cross-institution environmental prediction capacity. This structure is designed to provide the opportunity for (i) exploration and further insight into key cross-domain processes, (ii) a transdisciplinary work environment for participatory modelling, (iii) and the continual development and provision of tools across the whole decision-making workflow, resulting in (iv) outputs that are defensible and reproducible. By employing an adaptive approach, the proposed infrastructure will be responsive to technological and environmental changes and imperatives in the long term.

The framework proposed is informed by several federations around the globe including the National Centers for Environmental Prediction in the USA and the UK Environmental Prediction research project. The capability we are proposing for Australia is broader in scope than these, which are focused on particular areas of concern, such as risk from extreme weather events. There is growing impetus to develop such capabilities for broader environmental and ecological prediction, for example through the Ecological Forecasting Initiative in the USA, where NEON data are being leveraged to better assess short-term forecasting for ecological systems. We propose a framework that (i) will leverage the data from the NCRIS facilities so it can easily be incorporated into modelling systems, (ii) will provide an in-house, curated modelling capacity, and (iii) provide validation and visualisation opportunities to best enable predictions. These components will be coupled with a structure to support collaborative work with the wider research and policy-making communities not only to ensure there is effective, high quality inter- and trans-disciplinary input on subjects of concern, but to ensure relevant participatory modelling is facilitated, and model validation is active and open. Informed by such collaboration, relevant decision support tools will be accessed and made available to quickly test predictions about the effects of events such as wildfire.

We argue that six components need to be in place, interlocked and interdependent for the development of a responsive and adaptive framework:

1. readily available, high quality multi-domain data;

2. relevant data harmonised and scaled while ensuring provenance, vocabularies and workflows are properly described, registered and reproducible, ready for complex modelling;

3. a shared platform for the creation, harnessing and modification of models by users;

4. strong coordination with the wider community using an adaptive co-learning and co-design approach;

5. translation tools for model testing and communication of outcomes; and

6. social and technical governance structures to transparently manage and coordinate the overall program and the infrastructure.

Our aim is to ensure adherence to the FAIR principles for research, and where possible, the outputs will be openly accessible and re-usable. Although in the first instance we expect the framework will be largely used by researchers in government and academia, we envision, once it is established, it will be available to other users on a cost-recovery basis.

\footnotetext{
${ }^{1}$ where 'Research infrastructure' includes the physical facilities, human resources and related services that are used by the research community to conduct research' (Research Infrastructure Review Final Report September 2015, https://bit.ly/2WAPczc, accessed 17 August 2021)
}

Keywords: Environmental prediction, framework, digital infrastructure, modelling, Data as a Service 
Specht et al., Enabling integrated modelling systems for environmental prediction at a national scale

\section{INTRODUCTION}

Effective prediction of the consequences of human actions and likely future natural events is essential for the welfare of communities and the sustainability of ecosystems and human infrastructure. As a result, a growing number of organisations around the world have established organised forecasting systems. Meteorological data are among the initial targets for the development of such systems, and organising an umbrella organisation to link the components for forecasting is the concern of the National Centers for Environmental Prediction in the USA (https://www.weather.gov/ncep/). In Australia, we have the Australian Community Climate and Earth System Simulator (https://bit.ly/2XO40eE). The NOAA Ecological Forecasting for coastal communities in the USA (https://bit.ly/37ZZgo9) combines sophisticated data gathering with modelling and computing capacities to improve the capability of the scientific and operational oceanography community to monitor and forecast environmental changes and hazardous events (Nichols and Wright, 2020). Human health and epidemiology are also early subjects for integrated predictive systems where complex modelling is required, such as the EPSRC Centre for Predictive Modelling in Healthcare (https://bit.ly/2W7YfrS). An extension into an integrated environmentally-based forecasting framework is emerging with, for example, the development of a high resolution regionally coupled prediction system for the United Kingdom (https://bit.ly/3g19zrg), and the NSFfunded Ecological Forecasting Initiative-Research Coordination Network's Forecasting Challenge using NEON data (https://bit.ly/3sAz0Km). Integrated and responsive prediction systems founded on the increasing wealth of well-curated diverse data, sophisticated and complex modelling systems, virtual collaboration spaces, and simulation tools will go a long way towards ensuring a sustainable, resilient society.

\subsection{Australia}

A 2021 Australian government consultation about the need for a National Environmental Prediction System (NEPS) suggests that there are major shortcomings in Australia in our ability (i) to challenge model results with observations and experiments and vice versa, (ii) to predict results, (iii) to employ decision-making analytics, and (iv) to apply effective benchmarking. We propose that the transformation of ecosystem prediction into a rigorous, scientific endeavour can be made possible by better harnessing the already-existing scientific, human and technical capital across many scales, domains of knowledge, and spheres of concern (Figure 1). We have at our disposal (i) the extensive, well-curated historical and surveillance data of the NCRIS facilities (https://www.dese.gov.au/ncris), universities, research organisations and governments, (ii) the observing capacity of operational satellites from which Australia acquires data streams, (iii) the ample capacity of cloud computing and data storage (facilitated through the Australian Research Data Commons - ARDC),

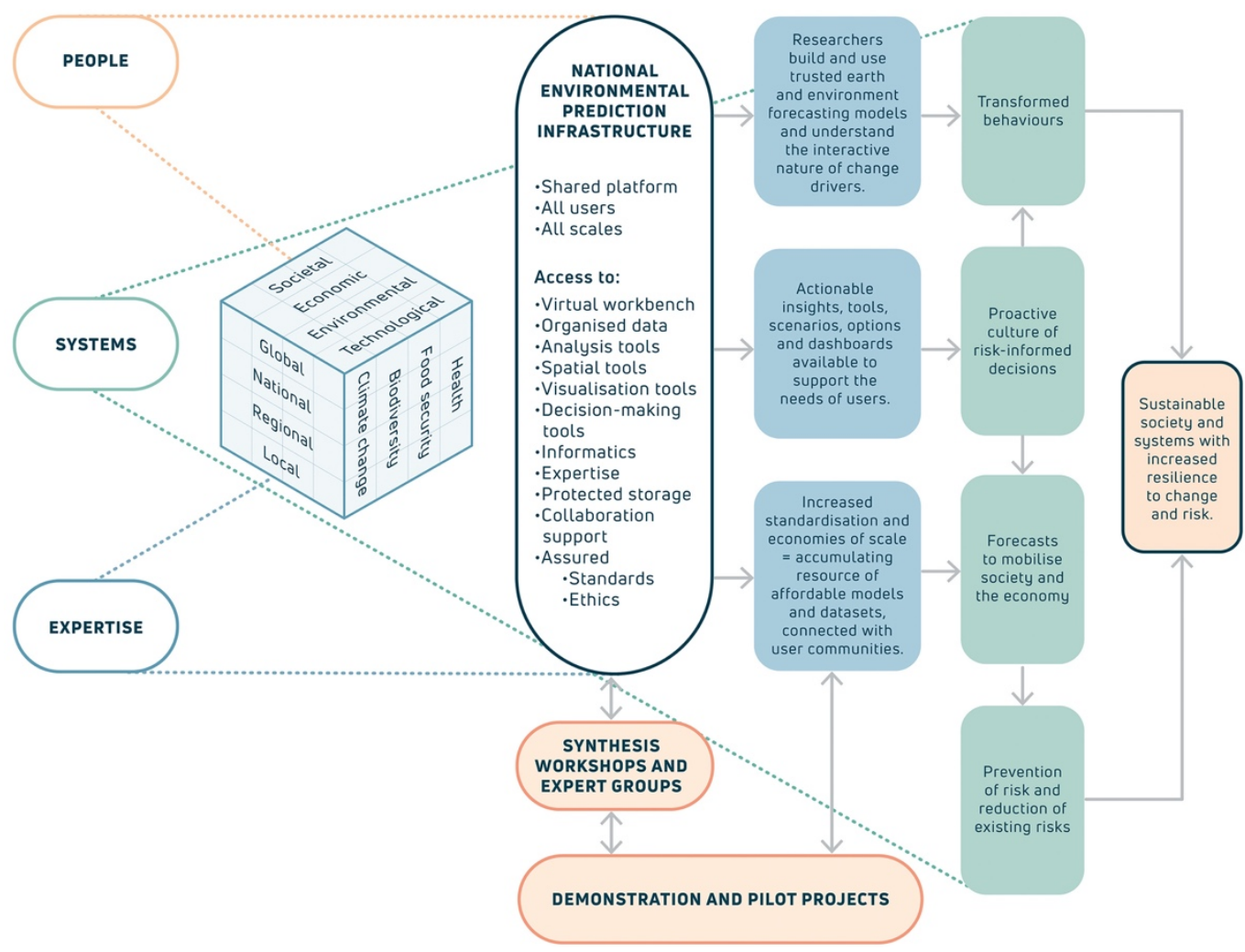

Figure 1. The context in which the national environmental prediction infrastructure will be situated and its anticipated effects. 
Specht et al., Enabling integrated modelling systems for environmental prediction at a national scale

(iv) the availability of high-performance computing (e.g. through the National Computational Infrastructure and the Pawsey Supercomputing Centre) for modelling of complex dynamic systems; (v) current advances in machine learning and artificial intelligence for mining and analysis of large quantities of data and, last but by no means least, (vi) the expertise of the whole Australian community. An infrastructure where this diverse technical and information capital can be critically reviewed by and blended with the expertise of the community in a supported environment, will enable the development of the complex, heterogeneous models required to predict future scenarios. Such a structure will provide continuing opportunity for the development of actionable insights, enhance our society's decision-making capability, create trust and collaborative skills between segments of the community, and increase our resilience to change and risk.

\subsection{Terrestrial Ecosystem Research Network (TERN)}

TERN has had ten years of experience responding to the needs of the ecosystem science community in building the infrastructure and the services associated with it. The on-going development of TERN was partly informed by a systematic survey in 2012 of 750 Australian ecologists and environmental scientists and managers in which it was found that $98 \%$ of respondents thought that interdisciplinary research collaboration was very important for modern ecosystem science and management, and $71 \%$ said that their research usually focused on multiple sets of questions or issues (making interdisciplinary research essential). Further, $68 \%$ felt that lack of access to data from other researchers or institutions was a major impediment to progress in science ( $19 \%$ were undecided). Most respondents found collaboration with others enjoyable and stimulating, but half worked with people from their own discipline or one close to theirs, and $64 \%$ of respondents only worked with collaborators from their own institution. Realising the potential for high quality interdisciplinary research clearly needed some assistance.

Since it became operational in 2010, TERN has monitored the requirements of researchers through multiple community consultations and committees associated with each of its infrastructure platforms. Between 2010 and 2015, TERN's Australian Centre for Ecological Analysis and Synthesis (ACEAS) brought together participants from research organisations and universities, all levels of government, NGOs and private consulting companies from around Australia and overseas to work together in 39 small groups (10-15 people on average) around complex multi-disciplinary problems. This exercise provided TERN with unique insight into the dynamics of inter- and trans-disciplinary work, and the infrastructure required to support it (Specht et al., 2015). Support was found to fall into four main categories: (i) assistance to acquire and organise a range of disparate, often very large, data sets, (ii) support and dedicated space to analyse and model the collated data, (iii) visualisation of the data to test the results for veracity and as a communication tool, and (iv) tools for policy transferability.

This support was rewarded by new insights and knowledge of possibilities through such collaboration, for example (with unique codes for ACEAS group feedback) 'I have learned that remote sensing has the potential to be used as a surrogate or predictor of atmospheric pollen levels' (DAVWS211), and 'this considerably widened my knowledge of possible technologies' (SPEWS112), and also identified gaps such as 'a need for instrument installation protocols' (PHEWS108), 'The need for a solution that will couple data discovery and processing in a consistent way' (SPEWS101), the need to 'Develop a map portal for display of elicitation results' (MCAWS214), and 'The possibility of using games to improve insights into values-placed ecosystems' (COSWS102). Participants in the ACEAS experiment relied on face-to-face meetings which all considered optimal, but the provision of '... "virtual meeting" tools might be useful' (MALWS102, MCAWS210). As virtual meeting tools have greatly improved and usage has spread in the community in recent years, this is now a more favourable option than it was at the time (2010-2015).

Informed by this experience and the feedback obtained from the ongoing co-design of the platforms within TERN, this paper presents a description of an infrastructure that will enable the highest degree of predictive analysis to be supported in Australia.

\section{THE PROPOSED FRAMEWORK}

A research infrastructure cannot be static. To support the highest quality research, it needs to be adaptive, and responsive to the science, current issues, and advances in technology. This is no mean feat but can be achieved by embedding collaborative partnerships and adaptive learning in the design, a dynamic approach which capitalises on the wealth of available data and expertise and will make flexible and timely predictive capability possible.

\subsection{Framework Components}

The framework described in this paper consists of four main components (Figure 2):

1. A Synthesis Capability to enable all stakeholders to come together as a transdisciplinary community of practice around critical questions, supported by the infrastructure; 
Specht et al., Enabling integrated modelling systems for environmental prediction at a national scale

2. A Loosely Coupled Modelling Infrastructure to enable the synthesis capability and to interface with providers;

3. Provision of the Governance and Standards responsible for ensuring best practice against international criteria, including principles and procedures for curating and managing access to data sets and models; and

4. Project-based access by the wider community.

The operational features of this structure (A-E, Figure 2) are discussed in detail in the following sections.

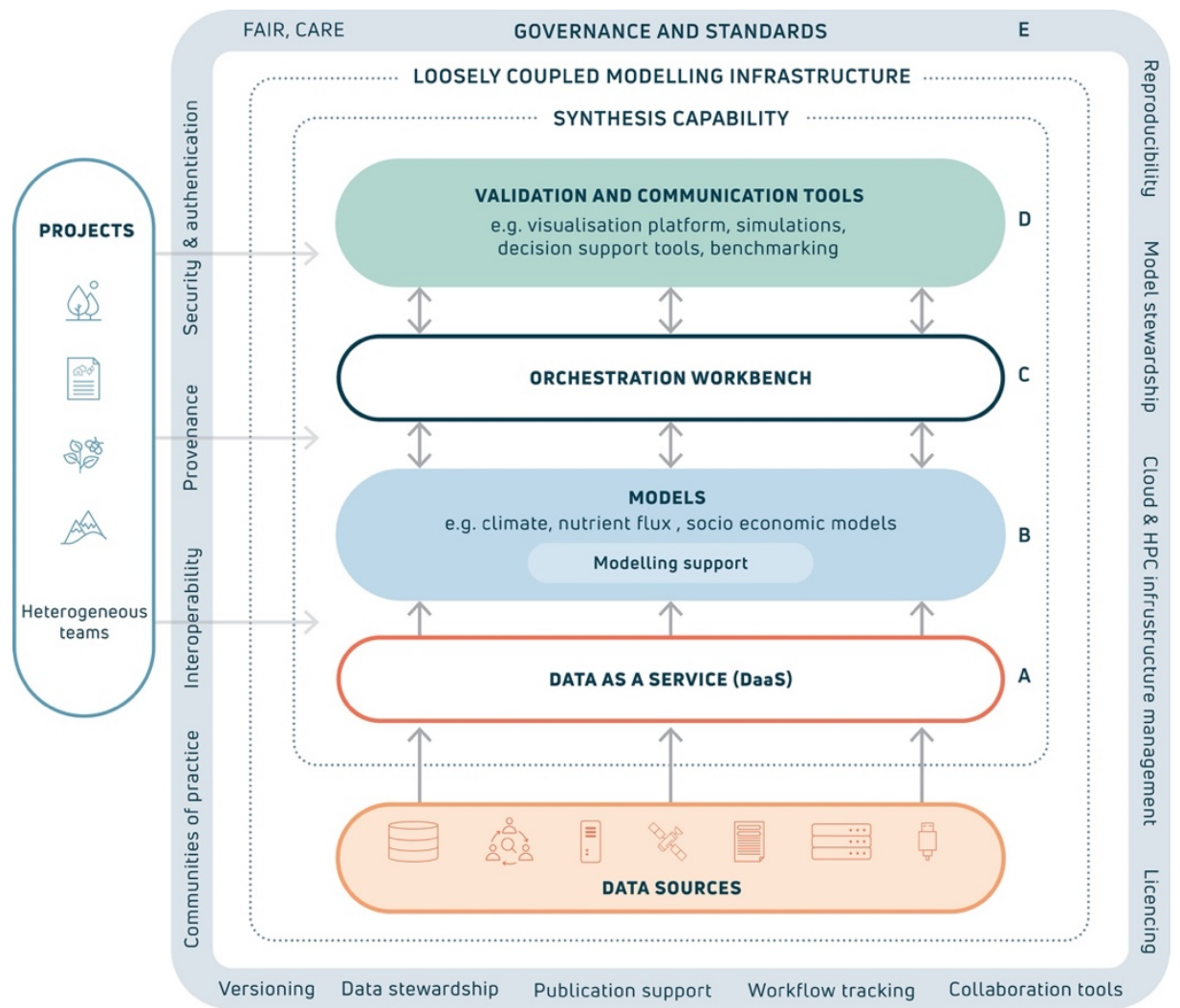

Figure 2. Components of the proposed framework to support an environmental prediction capability.

\subsubsection{Data as a Service (A)}

There are many data sources that can provide the base-line evidence for complex problem-solving, including government sources and the NCRIS stable of repositories, university servers, and laboratory or project records. Their nature can be highly variable. Most data repositories store data and associated metadata using domainspecific standards and provide access to data in a human-readable format. If each repository makes data fitfor-purpose for the needs of multiple models then there will be a multiple implementation of the data access interface and some of the interfaces may be vendor-specific. The accessibility of multiple interfaces with different specifications adds a layer of complexity in the model-data integration. The Data as a Service (DaaS) component of the framework (A, Figure 2) provides a centralised approach to the data management strategy to deliver data for integration and processing in a ready-to-use format and scale. The DaaS will improve data accessibility by implementing managed services to administer data in a structure and format to meet user needs. The DaaS enables the development of uniform services to access data with better data governance and leveraging cloud capability. With the DaaS, the system can provide service endpoints to meet the data needs of the models. With the capability to access data via an API, it will not be necessary to copy data locally for processing.

\subsubsection{Models (B)}

Developing and harnessing models are fundamental to forecasting change. Access to a wide variety of models in a reusable form is crucial for running complex prediction scenarios. A variety of existing models will be accessed, tested, linked and manipulated by team members in the Workbench (C, Figure 2). Users will be able to bring their own models to compare with existing models. Through this process, they will create new models and contribute to the improvement of existing ones. Models will be able to be tested through the validation provision (D, Figure 2). To facilitate model comparisons and modifications, elements of participatory modelling (Gray et al., 2018) and group model building (Chateau et al., 2012) will be among approaches used 
Specht et al., Enabling integrated modelling systems for environmental prediction at a national scale

to engage the complex groups involved. These types of approaches will help to bring a variety of expertise into prediction modelling and scenario building (Sang, 2020). To make them more reusable and accessible, models will be published with contextual metadata and will be discoverable.

\subsubsection{Orchestration Workbench $(C)$}

The Orchestration Workbench is the execution engine house which will enable collaborative work around complex, multi-dimensional problems. The Workbench is designed to be a virtual work platform allowing a team to access existing models relevant to the problem, and validation tools (such as visualisation and other scenario tools) and configure the execution pipeline in an interactive manner. Scientific workflows will be available through the Workbench to ensure each project's work is recorded from dataset selection and harmonisation (executed by the infrastructure services team) through to modelling, simulation and the development of new products useful for prediction. There are several work platforms available in Australia for this, such as CoESRA, which provides users with a storage and computational platform that is accessible from a web browser (Guru et al., 2016). One of the advantages of this system is that the majority of the tools needed by a group for their work can be incorporated, such as R and QGIS. In addition, protected environments can be created, allowing groups to work without scrutiny until they wish to share their results. With appropriate authentication, the system has few geographical limitations to participation, and certainly no storage challenges. The Orchestration Workbench will allow interaction with relevant virtual laboratories to execute specialised functionalities. It will use distributed resources, both computational and cloud storage, and high performance computing infrastructure. Provenance capturing is critical to verify, debug and reproduce model execution. Capturing all execution information in a distributed environment is a challenge, however, and requires further tool development to build cloud-aware provenance tracking.

\subsubsection{Validation and communication tools (D)}

Models need to be tested in a variety of ways as succinctly illustrated in Fer et al. (2021, Figure 2). The framework proposed will provide access to a variety of validation and communication tools, from spatial tools such as QGIS, to more complex decision support tools. Along with fundamental sensitivity and uncertainty analyses, visualisation is a key part of model testing and increasingly used in complete system simulations. System simulations, in as realistic a manner as possible, play an important role in empowering transdisciplinary research and co-design, and in the communication of results outside the working group itself. Although scenario testing and benchmarking with real-time or near real-time data is useful for the modellers as they refine their models and determine the uncertainty levels, prediction scenarios are particularly valuable for decision-makers. 'Predictions don't need to convince, but just get people thinking' (Steve Cork, p. 25 in Pirzl et al., 2019).

\subsubsection{Governance and standards (E)}

This section falls into two components, (i) monitoring the infrastructure, particularly the standards of the work conducted within it and the support provided for that work, and (ii) the logistic operation of the infrastructure and its interface with users and stakeholders.

Work within the infrastructure will be monitored to be consistent with international standards such as FAIR (https://www.go-fair.org/fair-principles/) and CARE (https://www.gida-global.org/care), and include all digital objects, including software and code (Lamprecht et al., 2020). CARE and FAIR incorporate many of the subjects listed separately in Figure 2 and will be ensured by the infrastructure team. This support is particularly important for working groups who spend only some of their time working on a problem. Although the framework proposed in this paper is not directed solely (or even principally) at enabling the production of peer reviewed articles, such articles in association with scenarios and horizon scanning outputs lend veracity to the product and help to benchmark it against international standards.

Active involvement by communities of practice across all domains is vital. This will be accomplished through a project-based approach. As illustrated in section 1.2, TERN has a track record of successful engagement with communities of practice. The guidelines presented in Lynch et al. (2015) to facilitate effective collaborations will be used to ensure optimal results. These guidelines include: (i) at least one face-to-face meeting per group (face to face meetings remain a core part of developing the trust that allows effective data and information sharing (Srivastava et al., 2021)), (ii) support for a prolonged period of interaction, following an agreed work plan, (iii) support for engagement with leading researchers and experts from science, policy and management sectors, (iv) informatics and research support (A-C, Figure 2), and (v) support for novel product delivery (D, Figure 2).

Sharing data, models and intellectual property requires a great deal of trust between parties. To be successful, the quality of the materials used as well as the conduct of the participants need to be trustworthy. Participants themselves need to be informed of the standards of appropriate conduct when in the working space. This is arguably more important when working remotely. They should adhere, for example, to the appropriate 
Specht et al., Enabling integrated modelling systems for environmental prediction at a national scale

standards of conduct consistent with the European Code of Conduct for Research Integrity (ALLEA, 2020). In practice this would include a signed 'code of conduct'. For inter- and transdisciplinary work to live up to its promise, the full participation of all members is necessary, which may require active management by the infrastructure team (Zscheischler and Rogga, 2015). Security of the working space and the data and models used is essential. There may be caveats and licences on sensitive data which may include the level of disclosure of human subject data. There may be restrictions around the geo-locations of rare species, and there may be caveats about use of bio-cultural data. Sometimes these conditions may eliminate the data from use entirely. The infrastructure staff may have to anonymise data or work on a solution to allow use. Active management of the cloud and HPC infrastructure and data and model stewardship not only require in-house management but also liaison with data providers, other HPC and cloud systems, and modelling and tool providers.

Overall quality assurance will be the responsibility of a governance committee which will monitor the scientific and technical support being provided by the infrastructure and its general conduct. This committee, with two sub-committees, one to support and guide the modelling and scenario development, and one for the data and HPC infrastructure, will include representatives from major stakeholders and jurisdictions and ensure that the infrastructure operates according to the best national and international standards of probity.

\subsubsection{Projects}

The infrastructure will be open to the scientific, government, non-government, citizen and industry communities. There will be some seed-funded opportunities which will be competitively granted as an incentive program, but it is envisioned that any group will be able to access the infrastructure if they (i) present a work plan consistent with the infrastructure aims of interdisciplinary, inter-organisational environmental predictive scenario development, and (ii) are able to fund the costs of their work plan. Use of the infrastructure by interested groups will be determined on a case-by-case basis, and general service costs will be published. The default for the infrastructure will be eventual disclosure of the source data, model and software codes, and access to the scenarios developed according to the most open Creative Commons licences (CC BY 4.0 will be the basic standard). It is anticipated that this will not be applicable in all situations. The incentive program envisaged will allow parties, such as government or industry, separately or together, to fund an initiative to solve a problem of concern, with teams submitting proposals that will be selected on merit. The Ecological Forecasting Challenge funded by the EFI-Research Coordination Network (EFI-RCN) is one example of how this might be done.

In the first instance, seed-funded projects would be identified in a structured manner by the wider community and stakeholders around key challenges for environmental prediction. Teams of people will be invited to tender to do the work, and a subset of the governance committee will be tasked with selection against published criteria. It is envisaged that these groups will interact with the infrastructure at several levels, but their work will be largely in the synthesis envelope in Figure 2, supported actively by the infrastructure support team. These projects, and those that are anticipated to follow, will provide a continuous learning opportunity for the staff of the infrastructure, as models are improved, data streams developed, external standards change, and new tools are discovered and implemented. This will ensure the infrastructure is sensitive to the needs of the users, and that its capability will be continually improved.

\section{CONCLUSION}

Establishing a capability in environmental prediction at a national level is a complex undertaking, requiring cross-domain and cross-sectoral integration, creation of models, and testing of scenarios. This is vital for us to have better control of our future, reduce and prevent risk, and sustain our environment to ensure the well being of society and the systems that support it.

Six components need to be in place, interlocked and interdependent for the development of a responsive and adaptive framework:

7. readily available, high quality multi-domain data;

8. relevant data harmonised and scaled while ensuring provenance, vocabularies and workflows are properly described, registered and reproducible, ready for complex modelling;

9. a shared platform for the creation, harnessing and modification of models by users;

10. strong coordination with the wider community using an adaptive co-learning and co-design approach;

11. translation tools for model testing and communication of outcomes; and

12. social and technical governance structures to transparently manage and coordinate the overall program and the infrastructure.

We have described a possible framework that will deliver these components in an integrated manner and enable efficient and effective collaboration across disciplinary and organisational space and support robust, innovative, outcomes. Through this process the people, systems, and expertise be brought together in a mutually adaptive way. 
Specht et al., Enabling integrated modelling systems for environmental prediction at a national scale

\section{REFERENCES}

Château, P.-A., Chang, Y.-C., Chen, H., Ko, T.-T., 2012. Building a stakeholder's vision of an offshore wind-farm project: A group modeling approach. Science of The Total Environment 420, 43-53. https://doi.org/10.1016/j.scitotenv.2012.01.031

ALLEA, 2020. European Conduct for Research Integrity, Revised Edition https://ec.europa.eu/info/fundingtenders/opportunities/docs/2021-2027/horizon/guidance/european-code-of-conduct-for-researchintegrity horizon en.pdf (accessed 21 August 2021)

Fer, I., Gardella, A.K., Shiklomanov, A.N., Campbell, E.E., Cowdery, E.M., De Kauwe, M.G., Desai, A., Duveneck, M.J., Fisher, J.B., Haynes, K.D., Hoffman, F.M., Johnston, M.R., Kooper, R., LeBauer, D.S., Mantooth, J., Parton, W.J., Poulter, B., Quaife, T., Raiho, A., Schaefer, K., Serbin, S.P., Simkins, J., Wilcox, K.R., Viskari, T., Dietze, M.C., 2021. Beyond ecosystem modeling: A roadmap to community cyberinfrastructure for ecological data-model integration. Glob. Change Biol. 27, 13-26. https://doi.org/10.1111/gcb.15409

Gray, S., Voinov, A., Paolisso, M., Jordan, R., BenDor, T., Bommel, P., Glynn, P., Hedelin, B., Hubacek, K., Introne, J., Kolagani, N., Laursen, B., Prell, C., Schmitt Olabisi, L., Singer, A., Sterling, E., Zellner, M., 2018. Purpose, processes, partnerships, and products: four Ps to advance participatory socioenvironmental modeling. Ecol Appl 28, 46-61. https://doi.org/10.1002/eap.1627

Guru, S., Hanigan, I.C., Nguyen, H.A., Burns, E., Stein, J., Blanchard, W., Lindenmayer, D., Clancy, T., 2016. Development of a cloud-based platform for reproducible science: A case study of an IUCN Red List of Ecosystems Assessment. Ecological Informatics 36, 221-230. https://doi.org/10.1016/j.ecoinf.2016.08.003

Lamprecht, A.-L., Garcia, L., Kuzak, M., Martinez, C., Arcila, R., Martin Del Pico, E., Dominguez Del Angel, V., van de Sandt, S., Ison, J., Martinez, P.A., McQuilton, P., Valencia, A., Harrow, J., Psomopoulos, F., Gelpi, J.Ll., Chue Hong, N., Goble, C., Capella-Gutierrez, S., 2020. Towards FAIR principles for research software. Data Science 3, 37-59. https://doi.org/10.3233/DS-190026

Lynch, A.J.J., Thackway, R., Specht, A., Beggs, P.J., Brisbane, S., Burns, E.L., Byrne, M., Capon, S.J., Casanova, M.T., Clarke, P.A., Davies, J.M., Dovers, S., Dwyer, R.G., Ens, E., Fisher, D.O., Flanigan, M., Garnier, E., Guru, S.M., Kilminster, K., Locke, J., Mac Nally, R., McMahon, K.M., Mitchell, P.J., Pierson, J.C., Rodgers, E.M., Russell-Smith, J., Udy, J., Waycott, M., 2015. Transdisciplinary synthesis for ecosystem science, policy and management: The Australian experience. Science of The Total Environment 534, 173-184. https://doi.org/10.1016/j.scitotenv.2015.04.100

National Environment Prediction System Scoping Study Discussion Paper, 2021. https://2021 nriroadmap.dese.gov.au/get-involved/scoping-study-consultation/discussion-paper-nepsscoping-study/ (accessed 31 August 2021).

Nichols, C.R., Wright, L.D., 2020. The Evolution and Outcomes of a Collaborative Testbed for Predicting Coastal Threats. JMSE 8, 612. https://doi.org/10.3390/jmse8080612

Pirzl, R., Doerr, V., Lemon, D., McDonald, W., Ahmad, M., Grigg, N., Fletcher, C., Mokany, K., Schandl, H., O’Connell, D., Palmer, R., Woodward, E. 2019. Environmental Prediction Symposium Synthesis. CSIRO Land and Water, Australia

Sang, N.S. (Ed.), 2020. Modelling Nature-based Solutions: Integrating Computational and Participatory Scenario Modelling for Environmental Management and Planning, 1st ed. Cambridge University Press. https://doi.org/10.1017/9781108553827

Specht, A., Guru, S., Houghton, L., Keniger, L., Driver, P., Ritchie, E.G., Lai, K., Treloar, A., 2015. Data management challenges in analysis and synthesis in the ecosystem sciences. Science of The Total Environment 534, 144-158. https://doi.org/10.1016/j.scitotenv.2015.03.092

Srivastava, D.S., Winter, M., Gross, L.J., Metzger, J.P., Baron, J.S., Mouquet, N., Meagher, T.R., Halpern, B.S., Pillar, V.D., 2021. Maintaining momentum for collaborative working groups in a post-pandemic world. Nature Ecology and Evolution 1-2. https://doi.org/10.1038/s41559-021-01521-0

Zscheischler, J., Rogga, S., 2015. Transdisciplinarity in land use science - A review of concepts, empirical findings and current practices. Futures 65, 28-44. https://doi.org/10.1016/j.futures.2014.11.005 